QUANTUM PROBABILITY

BANACH CENTER PUBLICATIONS, VOLUME 73

INSTITUTE OF MATHEMATICS

POLISH ACADEMY OF SCIENCES

WARSZAWA 2006

\title{
THE MONOTONE POISSON PROCESS
}

\author{
ALEXANDER C. R. BELTON \\ Institut Girard Desargues, Université Claude Bernard Lyon 1 \\ 43 avenue du 11 novembre 1918, Villeurbanne 69622 Cedex, France \\ E-mail: belton@igd.univ-lyon1.fr
}

\begin{abstract}
The coefficients of the moments of the monotone Poisson law are shown to be a type of Stirling number of the first kind; certain combinatorial identities relating to these numbers are proved and a new derivation of the Cauchy transform of this law is given. An investigation is begun into the classical Azéma-type martingale which corresponds to the compensated monotone Poisson process; it is shown to have the chaotic-representation property and its sample paths are described.
\end{abstract}

1. Introduction. If $X$ is a random variable distributed according to the monotone Poisson law, i.e., Muraki's monotonic law of small numbers [16, Section 4], with mean $t>0$ then $[4$, Section 4$]$

$$
\mathbb{E}\left[X^{n+1}\right]=t+\sum_{m=1}^{n}\left(\sum_{2 \leqslant j_{1}<\cdots<j_{m} \leqslant n+1} j_{1} \cdots j_{m}\right) \frac{t^{m+1}}{(m+1) !} \quad \forall n \geqslant 1 .
$$

The coefficients appearing in (1) are shown below to be a type of generalized Stirling number. Using a method of proof from our earlier work, closed forms for exponential generating functions of such Stirling numbers are derived; it seems these have appeared previously in the literature only for sporadic examples [19]. The Cauchy transform for the monotone Poisson law, found by Muraki in [16], is derived in a novel manner from the recurrence relation satisfied by the moment coefficients.

In [4] the monotone Poisson process was realised as a vacuum-adapted semimartingale; the corresponding regular quantum semimartingale, i.e., its HP-adapted projection [3], is used to construct a classical semimartingale $y=\left(y_{t}\right)_{t \geqslant 0}$ distributed according to the monotone Poisson law. It is shown that $y_{t}=x_{t}+t$ for all $t \geqslant 0$, where $x$ is a normal martingale (the compensated monotone Poisson process) which satisfies the structure equation

$$
x_{0}=0, \quad \mathrm{~d}[x]_{t}=\left(1-t-x_{t-}\right) \mathrm{d} x_{t}+\mathrm{d} t \quad \forall t \geqslant 0 .
$$

2000 Mathematics Subject Classification: Primary 46L53; Secondary 11B73, 60G44, 81S25.

The paper is in final form and no version of it will be published elsewhere. 
This martingale $x$ is shown to have the chaotic-representation property and to be purely discontinuous, with sample paths as follows: if $g_{t}:=\sup \left\{s \in[0, t]: x_{s}=1-s\right\}$ is the last time up to $t$ that $x_{s}=1-s$ then

$$
x_{t}=-W_{\bullet}\left(-\exp \left(-1-t+g_{t}\right)\right)-t \quad \forall t \geqslant 0,
$$

where $W_{\bullet}$ is one of the two real branches of the Lambert $W$ function (i.e., the multivalued inverse to the function $z \mapsto z e^{z}$ ). A numerical simulation is employed to produce approximations to these paths.

1.1. Notation. The set of natural numbers is denoted by $\mathbb{N}$ and equals $\{1,2,3, \ldots\}$; the set of non-negative integers is denoted by $\mathbb{Z}_{+}$and equals $\{0,1,2, \ldots\}$. If $\left\{f_{\alpha}: \alpha \in A\right\}$ is a collection of real-valued functions with common domain $\Omega$ then $\sigma\left(f_{\alpha}: \alpha \in A\right)$ is the smallest $\sigma$-algebra on $\Omega$ to make each function measurable. If $A$ is a subset of some algebra then the subalgebra that it generates is denoted by $\operatorname{alg} A$.

1.2. Conventions. An empty sum equals 0; an empty product equals 1. A function $f: A \rightarrow B$ has domain $\operatorname{Dom} f=A$ and range $\operatorname{Ran} f=f(A) \subseteq B$. The binomial coefficient $\left(\begin{array}{c}n \\ m\end{array}\right)$ equals 0 if $m<0$.

\section{A recurrence relation}

Definition 1. For all $a \in \mathbb{Z}_{+}$define

$$
||_{a}: \mathbb{N} \times \mathbb{N} \rightarrow \mathbb{Z}_{+} ;(n, m) \mapsto\left|\begin{array}{c}
n \\
m
\end{array}\right|_{a}
$$

by setting $\left|\begin{array}{l}n \\ 1\end{array}\right|_{a}=1$ for all $n \in \mathbb{N},\left|\begin{array}{c}n \\ m\end{array}\right|=0$ if $m>n$ and by using the recurrence relation

$$
\left|\begin{array}{c}
n+1 \\
m+1
\end{array}\right|_{a}=\left|\begin{array}{c}
n \\
m+1
\end{array}\right|_{a}+(n-1+a)\left|\begin{array}{c}
n \\
m
\end{array}\right|_{a} \quad \forall n, m \in \mathbb{N}
$$

These numbers are generalized Stirling numbers of the first kind; this terminology is justified by Remark 5 and Remark 6 below.

Proposition 2. The function ||$_{a}$ is well defined for all $a \in \mathbb{Z}_{+}$and satisfies

$$
\left|\begin{array}{l}
n \\
n
\end{array}\right|_{a}=\prod_{j=0}^{n-2}(j+a)=\left\{\begin{array}{cc}
\delta_{1}^{n} & \text { if } a=0 \\
(n-2+a) ! /(a-1) ! & \text { if } a \in \mathbb{N}
\end{array}\right.
$$

for all $n \in \mathbb{N}$, where $\delta$ is the Kronecker delta ( $\delta_{b}^{a}=1$ if $a=b$ and 0 otherwise).

Proof. Induction.

The recurrence relation (3) and the boundary conditions provide a quick method for the computation of ||$_{a}$; a range of values for $\left.\right|_{2}$ is given in Table 1 .

Proposition 3.

$$
\left|\begin{array}{c}
n+1 \\
m+1
\end{array}\right|_{a}=\sum_{a \leqslant j_{1}<\cdots<j_{m} \leqslant n-1+a} j_{1} \cdots j_{m}
$$

for all $n, m \in \mathbb{N}$, where the summation is taken over integral $j_{1}, \ldots, j_{m}$. 


\begin{tabular}{r|rrrrrrrrrr}
$n^{m}$ & 1 & 2 & 3 & 4 & 5 & 6 & 7 & 8 & 9 & 10 \\
\hline 1 & 1 & 0 & 0 & 0 & 0 & 0 & 0 & 0 & 0 & 0 \\
2 & 1 & 2 & 0 & 0 & 0 & 0 & 0 & 0 & 0 & 0 \\
3 & 1 & 5 & 6 & 0 & 0 & 0 & 0 & 0 & 0 & 0 \\
4 & 1 & 9 & 26 & 24 & 0 & 0 & 0 & 0 & 0 & 0 \\
5 & 1 & 14 & 71 & 154 & 120 & 0 & 0 & 0 & 0 & 0 \\
6 & 1 & 20 & 155 & 580 & 1044 & 720 & 0 & 0 & 0 & 0 \\
7 & 1 & 27 & 295 & 1665 & 5104 & 8028 & 5040 & 0 & 0 & 0 \\
8 & 1 & 35 & 511 & 4025 & 18424 & 48860 & 69264 & 40320 & 0 & 0 \\
9 & 1 & 44 & 826 & 8624 & 54649 & 214676 & 509004 & 663696 & 362880 & 0 \\
10 & 1 & 54 & 1266 & 16884 & 140889 & 761166 & 2655764 & 5753736 & 6999840 & 3628800
\end{tabular}

Table $1 .\left|\begin{array}{c}n \\ m\end{array}\right|_{2}$ for $n, m=1, \ldots, 10$.

Proof. Define $f_{a}: \mathbb{N} \times \mathbb{N} \rightarrow \mathbb{Z}_{+}$by setting $f_{a}(n, 1)=1$ for all $n \in \mathbb{N}$ and

$$
f_{a}(n+1, m+1)=\sum_{a \leqslant j_{1}<\cdots<j_{m} \leqslant n-1+a} j_{1} \cdots j_{m} \quad \forall n, m \in \mathbb{N}
$$

note that $f_{a}(n, m)=0$ if $m>n$. Furthermore, if $n, m \in \mathbb{N}$ are such that $2 \leqslant m \leqslant n$ then

$$
\begin{aligned}
f_{a}(n+1, m+1) & =\sum_{a \leqslant j_{1}<\cdots<j_{m} \leqslant n-2+a} j_{1} \cdots j_{m}+\sum_{a \leqslant j_{1}<\cdots<j_{m-1} \leqslant n-2+a} j_{1} \cdots j_{m-1}(n-1+a) \\
& =f_{a}(n, m+1)+(n-1+a) f_{a}(n, m) ;
\end{aligned}
$$

this identity also holds if $m>n$ or $m=1$ :

$$
f_{a}(n+1, m+1)=0=0+0=f_{a}(n, m+1)+f_{a}(n, m) \quad \forall m>n
$$

and

$$
f_{a}(n+1,2)=\sum_{j_{1}=a}^{n-1+a} j_{1}=f_{a}(n, 2)+(n-1+a)=f_{a}(n, 2)+(n-1+a) f_{a}(n, 1) .
$$

Thus $f_{a}$ and ||$_{a}$ both satisfy the recurrence relation (3), with the same boundary conditions; the result follows.

COROllary 4. If $X$ is a random variable with the monotone Poisson distribution and mean $t>0$ then

$$
\mathbb{E}\left[X^{n}\right]=\sum_{m=1}^{\infty}\left|\begin{array}{l}
n \\
m
\end{array}\right|_{2} \frac{t^{m}}{m !} \quad \forall n \in \mathbb{N} .
$$

Proof. This is immediate from Proposition 3 and (1).

The definition of ||$_{2}$ extends to $\mathbb{Z}_{+} \times \mathbb{Z}_{+}$by setting $\left|\begin{array}{l}0 \\ 0\end{array}\right|_{2}=1$ and $\left|\begin{array}{l}n \\ 0\end{array}\right|_{2}=\left|\begin{array}{l}0 \\ n\end{array}\right|_{2}=0$ for all $n \in \mathbb{N}$; the identity (3) holds for all $n, m \in \mathbb{Z}_{+}$and, in the notation of Corollary 4 ,

$$
\mathbb{E}\left[X^{n}\right]=\sum_{m=0}^{\infty}\left|\begin{array}{l}
n \\
m
\end{array}\right|_{2} \frac{t^{m}}{m !} \quad \forall n \in \mathbb{Z}_{+} .
$$


REMARK 5. By Proposition 3, if $n \in \mathbb{N}$ then

$$
\prod_{j=0}^{n-1}(x+j+a)=x^{n}+\sum_{m=1}^{n}\left(\sum_{a \leqslant j_{1}<\cdots<j_{m} \leqslant n-1+a} j_{1} \cdots j_{m}\right) x^{n-m}=\sum_{m=0}^{n}\left|\begin{array}{c}
n+1 \\
m+1
\end{array}\right| x_{a} x^{n-m} .
$$

Hence, in the notation of Mitrinović [15] (who appears to have been the first to study such numbers),

$$
\left|\begin{array}{c}
n+1 \\
m+1
\end{array}\right|_{a}=R_{n}^{n-m}(-a,-1)=(-1)^{n-m} R_{n}^{n-m}(a, 1) \quad \forall n \in \mathbb{N}, m=0, \ldots, n .
$$

In terms of Broder's $r$-Stirling numbers of the first kind [5],

$$
\left|\begin{array}{c}
n+1 \\
m+1
\end{array}\right|_{a}=\left[\begin{array}{c}
n+a \\
n+a-m
\end{array}\right]_{a} \quad \forall n, m \in \mathbb{Z}_{+}
$$

and, in the unified approach of Hsu and Shiue [12],

$$
\left|\begin{array}{c}
n+1 \\
m+1
\end{array}\right|_{a}=S^{1}(n, n-m ;-1,0, a)=R_{1}(n, n-m, a) \quad \forall n \in \mathbb{Z}_{+}, m=0, \ldots, n,
$$

where $R_{1}(n, k, \lambda)$ is the $r$-Stirling polynomial of the first kind due to Carlitz $[6,7]$.

REMARK 6. If [ ] denotes the unsigned Stirling numbers of the first kind, following Karamata via Graham, Knuth and Patashnik [10], then

$$
\left.\left.\left[\begin{array}{c}
n \\
m
\end{array}\right]=\left|\begin{array}{c}
n+1 \\
n+1-m
\end{array}\right|_{0} \quad \forall n \in \mathbb{Z}_{+}, m \in \mathbb{Z} \cap\right]-\infty, n\right] .
$$

Gupta [11, Chapter 5] introduces numbers $G(n, m)$ such that $G(n, m)=\left|\begin{array}{c}n+1 \\ m+1\end{array}\right|_{1}$ for all $n, m \in \mathbb{Z}_{+}$. Since

$\sum_{m=0}^{n+1}\left|\begin{array}{c}n+2 \\ m+1\end{array}\right|_{0} x^{n+1-m}=\prod_{j=0}^{n}(x+j)=x \prod_{j=0}^{n-1}(x+j+1)=\sum_{m=0}^{n}\left|\begin{array}{c}n+1 \\ m+1\end{array}\right|_{1} x^{n+1-m} \quad \forall n \in \mathbb{N}$, $\left|\begin{array}{c}n+2 \\ m+1\end{array}\right|_{0}=\left|\begin{array}{c}n+1 \\ m+1\end{array}\right|_{1}$ for all $n \in \mathbb{N}$ and all $m \in \mathbb{Z}_{+}$with $m \leqslant n$. It is readily verified that this identity holds for all $n, m \in \mathbb{Z}_{+}$and

$$
G(n, m)=\left|\begin{array}{c}
n+1 \\
m+1
\end{array}\right|_{1}=\left|\begin{array}{c}
n+2 \\
m+1
\end{array}\right|_{0}=\left[\begin{array}{c}
n+1 \\
n+1-m
\end{array}\right],
$$

i.e., Gupta's numbers are unsigned Stirling numbers of the first kind.

A result of Mitrinović [15, (1.4)] implies that

$$
\left|\begin{array}{c}
n \\
m
\end{array}\right|_{a}=\sum_{j=0}^{m-1}\left|\begin{array}{c}
n \\
m-j
\end{array}\right|_{0}\left(\begin{array}{c}
n-m+j \\
n-m
\end{array}\right) a^{j} \quad \forall a \in \mathbb{Z}_{+} .
$$

The following transformation theorem is a generalization of this result.

TheOREM 7. If $a, b \in \mathbb{Z}_{+}$then

$$
\left|\begin{array}{c}
n \\
m
\end{array}\right|_{a}=\sum_{j=0}^{m-1}\left|\begin{array}{c}
n \\
m-j
\end{array}\right|_{b}\left(\begin{array}{c}
n-m+j \\
n-m
\end{array}\right)(a-b)^{j} \quad \forall n, m \in \mathbb{N} .
$$


Proof. If $n \in \mathbb{N}$ then, by Remark 5,

$$
\prod_{j=0}^{n-1}(x+j+b)=\sum_{m=0}^{n}\left|\begin{array}{c}
n+1 \\
m+1
\end{array}\right|_{b} x^{n-m}=\sum_{l=0}^{n}\left|\begin{array}{c}
n+1 \\
n+1-l
\end{array}\right|_{b} x^{l}
$$

and so, since $x+j+a=(x+a-b)+j+b$,

$$
\begin{aligned}
\prod_{j=0}^{n-1}(x+j+a) & =\sum_{l=0}^{n}\left|\begin{array}{c}
n+1 \\
n+1-l
\end{array}\right|_{b}(x+a-b)^{l} \\
& =\sum_{l=0}^{n} \mid \begin{array}{c}
n+1 \\
n+1-l \mid
\end{array} \sum_{m=0}^{l}\left(\begin{array}{c}
l \\
m
\end{array}\right)(a-b)^{l-m} x^{m} \\
& =\sum_{m=0}^{n} \sum_{l=m}^{n}\left|\begin{array}{c}
n+1 \\
n+1-l
\end{array}\right|_{b}\left(\begin{array}{c}
l \\
m
\end{array}\right)(a-b)^{l-m} x^{m} .
\end{aligned}
$$

Hence, if $m \in \mathbb{Z}_{+}$is such that $0 \leqslant m \leqslant n$,

$$
\begin{aligned}
\left|\begin{array}{c}
n+1 \\
n+1-m
\end{array}\right|_{a} & =\sum_{l=m}^{n}\left|\begin{array}{c}
n+1 \\
n+1-l
\end{array}\right|_{b}\left(\begin{array}{c}
l \\
m
\end{array}\right)(a-b)^{l-m} \\
& =\sum_{j=0}^{n-m}\left|\begin{array}{c}
n+1 \\
n+1-m-j
\end{array}\right|_{b}\left(\begin{array}{c}
m+j \\
m
\end{array}\right)(a-b)^{j}
\end{aligned}
$$

and the result follows. (The cases $m>n$ and $m=n=1$ are verified immediately upon inspection.)

REMARK 8. Theorem 7, with $b=0$, and Remark 6 yield the identities

$$
\left|\begin{array}{c}
n+k+1 \\
n+1
\end{array}\right|_{a}=\sum_{j=0}^{n}\left[\begin{array}{c}
n+k \\
j+k
\end{array}\right]\left(\begin{array}{c}
j+k \\
k
\end{array}\right) a^{j} \quad \forall n, k, a \in \mathbb{Z}_{+},
$$

which correspond, in the cases $a=2, \ldots, 4, k=1, \ldots, 4$ (and $k=5$ if $a=2$ ) to formulae [19, A001705-A001709, A001711-A001714, A001716-A001719, A001721-A001724] given by Crstici.

3. Generating functions. Recall that the exponential generating function for a sequence $\left(b_{n}\right)_{n \geqslant 0}$ is the function determined by the power series $\sum_{n=0}^{\infty} b_{n} z^{n} / n$ !.

Definition 9. For all $a \in \mathbb{Z}_{+}$define

$$
g_{a, n}(w):=\sum_{m=1}^{\infty}\left|\begin{array}{l}
n \\
m
\end{array}\right|_{a} \frac{w^{m}}{m !}=\sum_{m=1}^{n}\left|\begin{array}{l}
n \\
m
\end{array}\right|_{a} \frac{w^{m}}{m !} \quad \forall n \in \mathbb{N}, w \in \mathbb{C} .
$$

Note that $g_{a, n}(0)=0, g_{a, 1}(w)=w$ and

$$
g_{a, n+1}(w)=w+\sum_{m=2}^{\infty}\left|\begin{array}{c}
n+1 \\
m
\end{array}\right| \frac{w^{m}}{m !}=w+\sum_{m=2}^{\infty}\left|\begin{array}{c}
n \\
m
\end{array}\right| \frac{w^{m}}{m !}+(n-1+a) \sum_{m=2}^{\infty}\left|\begin{array}{c}
n \\
m-1
\end{array}\right| \frac{w^{m}}{m !},
$$

SO

$$
\left(g_{a, n+1}-g_{a, n}\right)^{\prime}=(n-1+a) g_{a, n} \quad \forall n \in \mathbb{N} .
$$


THEOREM 10. If $a \in \mathbb{N}$ and

$$
h_{a, n}(w, z):=\frac{1}{n !}\left(\frac{\partial}{\partial z}\right)^{n-1} \frac{(w-\log (1-z))^{n}}{(1-z)^{a}} \quad(w \in \mathbb{C},|z|<1)
$$

for all $n \in \mathbb{N}$ (where $\log$ denotes the principal branch of the natural logarithm) then $g_{a, n}(w)=h_{a, n}(w, 0)$ for all $n \in \mathbb{N}$ and all $w \in \mathbb{C}$.

Proof. Note first that $h_{a, 1}(w, 0)=w$ and $[10,(5.56)]$

$$
\frac{1}{(1-z)^{a}}=\sum_{l=0}^{\infty}\left(\begin{array}{c}
l+a-1 \\
a-1
\end{array}\right) z^{l} \quad(|z|<1)
$$

Next, letting $\partial_{z}^{m}:=\frac{\partial^{m}}{\partial z^{m}}$ et cetera,

$$
\begin{aligned}
n h_{a, n}(w, z) & =\frac{1}{(n-1) !} \sum_{m=0}^{n-1}\left(\begin{array}{c}
n-1 \\
m
\end{array}\right) \partial_{z}^{m}(w-\log (1-z))^{n} \partial_{z}^{n-1-m} \sum_{l=0}^{\infty}\left(\begin{array}{c}
l+a-1 \\
a-1
\end{array}\right) z^{l} \\
& =\sum_{m=0}^{n-1} \frac{1}{m !} j_{m, n}(w, z)\left(\left(\begin{array}{c}
n-m+a-2 \\
a-1
\end{array}\right)+(n-m)\left(\begin{array}{c}
n-m+a-1 \\
a-1
\end{array}\right) z+\cdots\right) \\
& =\sum_{m=0}^{n-1} \frac{(n-m+a-2) !}{m !(n-m-1) !} j_{m, n}(w, z)(1+(n-m+a-1) z+\cdots),
\end{aligned}
$$

where $j_{m, n}(w, z)=\partial_{z}^{m}(w-\log (1-z))^{n}$ and the ellipses denote a term of order $z^{2}$. If

$$
r_{a, n}:=\partial_{w}\left(h_{a, n+1}-h_{a, n}\right)-(n-1+a) h_{a, n}=\left(\partial_{z}-\partial_{w}-(n-1+a)\right) h_{a, n}
$$

then

$$
n r_{a, n}(w, 0)=\sum_{m=0}^{n-1} \frac{(n-m-2+a) !}{m !(n-m-1) !}\left(j_{m+1, n}(w, 0)-n j_{m, n-1}(w, 0)-m j_{m, n}(w, 0)\right)
$$

but $j_{m+1, n}(w, 0)=m j_{m, n}(w, 0)+n j_{m, n-1}(w, 0)[4$, Proof of Proposition 4.1] and so $r_{a, n}(w, 0)=0$ for all $w \in \mathbb{C}$ and all $n \in \mathbb{N}$. Thus the differential equation (4) is satisfied, with $g_{a, n}$ replaced by $w \mapsto h_{a, n}(w, 0)$ et cetera; as $h_{a, n}(0,0)=0$ for all $n \in \mathbb{N}$, because $j_{m, n}(0,0)=0$ if $0 \leqslant m<n$ [4, Proof of Proposition 4.1], the result follows.

Lemma 11. Given any $a, k \in \mathbb{Z}_{+}$not both zero, the power series

$$
\sum_{n=1}^{\infty}\left|\begin{array}{c}
n+k \\
n
\end{array}\right| \frac{z^{n}}{n !}
$$

has radius of convergence 1 . If $a=k=0$, the series (7) equals $z$.

Proof. If $a \in \mathbb{N}$ then, by Proposition 3,

$$
\left(\begin{array}{c}
n+k \\
n
\end{array}\right) \prod_{j=a}^{n-1+a} j \leqslant\left|\begin{array}{c}
n+1+k \\
n+1
\end{array}\right|_{a}=\sum_{a \leqslant j_{1}<\cdots<j_{n} \leqslant n+k-1+a} j_{1} \cdots j_{n} \leqslant\left(\begin{array}{c}
n+k \\
n
\end{array}\right) \prod_{j=k+a}^{n+k-1+a} j
$$

for all $k \in \mathbb{Z}_{+}$and all $n \in \mathbb{N}$, so if

$$
b_{n}:=\frac{1}{n !}\left(\begin{array}{c}
n-1+k \\
n-1
\end{array}\right) \prod_{j=a}^{n-2+a} j=\left(\begin{array}{c}
n-1+k \\
n-1
\end{array}\right) \frac{(n-2+a) !}{n !(a-1) !}
$$


and

$$
c_{n}:=\frac{1}{n !}\left(\begin{array}{c}
n-1+k \\
n-1
\end{array}\right) \prod_{j=k+a}^{n+k-2+a} j=\left(\begin{array}{c}
n-1+k \\
n-1
\end{array}\right) \frac{(n+k-2+a) !}{n !(k-1+a) !}
$$

then $b_{n} \leqslant \frac{1}{n !}\left|\begin{array}{c}n+k \\ n\end{array}\right| \leqslant c_{n}$ for all $n \in \mathbb{N}$; the result follows by the ratio and comparison tests. If $a=0$ then, since $\left|\begin{array}{c}n+2 \\ m+1\end{array}\right|_{0}=\left|\begin{array}{c}n+1 \\ m+1\end{array}\right|_{1}$ for all $n, m \in \mathbb{Z}_{+}$(Remark 6),

$$
\sum_{n=1}^{\infty}\left|\begin{array}{c}
n+k \\
n
\end{array}\right| \frac{z^{n}}{n !}=\sum_{n=1}^{\infty}\left|\begin{array}{c}
n+k-1 \\
n
\end{array}\right| \frac{z^{n}}{n !}
$$

has radius of convergence 1 for all $k \in \mathbb{N}$; the final case follows from Proposition 2.

The next result was proved by Broder [5, Theorem 15] using combinatorial methods. COROLlary 12. Given any $a, k \in \mathbb{N}$, the exponential generating function for the sequence $\left(b_{n}\right)_{n \geqslant 0}$, where $b_{0}=0$ and $b_{n}=\left|\begin{array}{c}n+k \\ n\end{array}\right|_{a}$ for all $n \in \mathbb{N}$, equals

$$
\frac{1}{k !}\left(\frac{\mathrm{d}}{\mathrm{d} z}\right)^{k-1} \frac{(-\log (1-z))^{k}}{(1-z)^{a}} \quad(|z|<1) .
$$

Proof. If $n, k \in \mathbb{N}$ then Theorem 10 implies that

$$
\begin{aligned}
\left|\begin{array}{c}
n+k \\
n
\end{array}\right|_{a} & =\left.\left(\frac{\partial}{\partial w}\right)^{n} \frac{1}{(n+k) !}\left(\frac{\partial}{\partial z}\right)^{n+k-1} \frac{(w-\log (1-z))^{n+k}}{(1-z)^{a}}\right|_{z=0, w=0} \\
& =\left.\frac{1}{(n+k) !}\left(\frac{\mathrm{d}}{\mathrm{d} z}\right)^{n+k-1} \frac{(n+k) !}{k !} \frac{(-\log (1-z))^{k}}{(1-z)^{a}}\right|_{z=0} \\
& =\left.\left(\frac{\mathrm{d}}{\mathrm{d} z}\right)^{n}\left(\frac{1}{k !}\left(\frac{\mathrm{d}}{\mathrm{d} z}\right)^{k-1} \frac{(-\log (1-z))^{k}}{(1-z)^{a}}\right)\right|_{z=0},
\end{aligned}
$$

as required.

Amusingly, Corollary 12 is also true for $k=0$, if $(\mathrm{d} / \mathrm{d} z)^{-1}$ is interpreted as integration. More precisely, if $a \in \mathbb{N}$ then, by Proposition 2 ,

$$
\left.\left.\sum_{n=1}^{\infty}\right|_{n} ^{n}\right|_{a} \frac{z^{n}}{n !}=\sum_{m=0}^{\infty}\left(\begin{array}{c}
m+a-1 \\
a-1
\end{array}\right) \int_{0}^{z} w^{m} \mathrm{~d} w=\int_{0}^{z} \frac{1}{(1-w)^{a}} \mathrm{~d} w
$$

where the second equality follows from (6). Hence

$$
\left.\left.\sum_{n=1}^{\infty}\right|_{n} ^{n}\right|_{a} \frac{z^{n}}{n !}=\left\{\begin{array}{cl}
z & \text { if } a=0 \\
-\log (1-z) & \text { if } a=1 \\
\frac{1}{a-1}\left(\frac{1}{(1-z)^{a-1}}-1\right) & \text { if } a \geqslant 2 .
\end{array}\right.
$$

Remark 13. By Remark 6, Corollary 12 and (8), if $k \geqslant 2$ then

$$
\sum_{n=1}^{\infty}\left|\begin{array}{c}
n+k \\
n
\end{array}\right| \frac{z^{n}}{n !}=\sum_{n=1}^{\infty}\left|\begin{array}{c}
n+k-1 \\
n
\end{array}\right|_{1} \frac{z^{n}}{n !}=\frac{1}{(k-1) !}\left(\frac{\mathrm{d}}{\mathrm{d} z}\right)^{k-2} \frac{(-\log (1-z))^{k-1}}{1-z}
$$




$$
\sum_{n=1}^{\infty}\left|\begin{array}{c}
n+1 \\
n
\end{array}\right|_{0} \frac{z^{n}}{n !}=\sum_{n=1}^{\infty}\left|\begin{array}{l}
n \\
n
\end{array}\right|_{1} \frac{z^{n}}{n !}=-\log (1-z) \quad \text { and } \quad \sum_{n=1}^{\infty}\left|\begin{array}{l}
n \\
n
\end{array}\right|_{0} \frac{z^{n}}{n !}=z .
$$

If $k \in \mathbb{Z}_{+}$then, by Remark 6 ,

$$
\sum_{n=1}^{\infty}\left|\begin{array}{c}
n+k \\
n
\end{array}\right| \frac{z^{n}}{n !}=\sum_{n=1}^{\infty}\left[\begin{array}{c}
n+k-1 \\
k
\end{array}\right] \frac{z^{n}}{n !}=\int_{0}^{z} \sum_{m=0}^{\infty}\left[\begin{array}{c}
m+k \\
k
\end{array}\right] \frac{w^{m}}{m !} \mathrm{d} w
$$

and so $[10,(7.50)]$

$$
\sum_{n=0}^{\infty}\left[\begin{array}{c}
n+k \\
k
\end{array}\right] \frac{z^{n}}{n !}=\frac{1}{k !}\left(\frac{\mathrm{d}}{\mathrm{d} z}\right)^{k}(-\log (1-z))^{k} \quad \forall k \in \mathbb{Z}_{+}
$$

4. A Cauchy transform. If $X$ is a random variable distributed according to the measure $\mu$ then the Cauchy transform (or Cauchy-Stieltjes transform) of $X$ is

$$
G_{X}: \mathbb{C} \backslash \operatorname{supp} \mu \rightarrow \mathbb{C} ; z \mapsto \int_{\operatorname{supp} \mu} \frac{\mathrm{d} \mu(x)}{z-x} .
$$

This function is holomorphic on $\mathbb{C} \backslash \operatorname{supp} \mu$ and, if $\operatorname{supp} \mu \subseteq[a, b]$,

$$
G_{X}(z)=\frac{1}{z} \int_{a}^{b} \sum_{n=0}^{\infty} \frac{x^{n}}{z^{n}} \mathrm{~d} \mu(x)=\sum_{n=0}^{\infty} \mathbb{E}\left[X^{n}\right] z^{-n-1} \quad(|z|>\max \{|a|,|b|\}) .
$$

LEMma 14. If $m \in \mathbb{N}$ then the series

$$
\sum_{n=1}^{\infty}\left|\begin{array}{l}
n \\
m
\end{array}\right|_{2} z^{-n-1}
$$

is absolutely convergent for all $z \in \mathbb{C}$ such that $|z|>1$ and, in that region, has sum

$$
F_{m}(z)=\left(\frac{z}{1-z} \frac{\mathrm{d}}{\mathrm{d} z}\right)^{m-1} \frac{1}{z(z-1)} .
$$

The double series

$$
\sum_{m=1}^{\infty} \sum_{n=1}^{\infty}\left|\begin{array}{l}
n \\
m
\end{array}\right|_{2} z^{-n-1} \frac{w^{m}}{m !}
$$

is absolutely convergent in $\left\{(z, w) \in \mathbb{C}^{2}:|z|^{1 / 2}>1+|w|^{1 / 2}\right\}$.

Proof. Since $\left|\begin{array}{l}n \\ 1\end{array}\right|_{2}=1$ for all $n \in \mathbb{N}$, the case $m=1$ is immediate. If $m \in \mathbb{N}$ then (working as in the proof of Lemma 11)

$$
\left(\begin{array}{c}
n \\
m
\end{array}\right) \frac{(m+1) !}{1 !} \leqslant\left|\begin{array}{c}
n+1 \\
m+1
\end{array}\right|_{2} \leqslant\left(\begin{array}{c}
n \\
m
\end{array}\right) \frac{(n+1) !}{(n+1-m) !} \quad \forall n \geqslant m
$$

and the ratio and comparison tests yield convergence as claimed. Furthermore,

$$
\begin{aligned}
F_{m+1}(z):=\sum_{n=m+1}^{\infty}\left|\begin{array}{c}
n \\
m+1
\end{array}\right|_{2} z^{-n-1} & =\sum_{n=m+1}^{\infty}\left(\left|\begin{array}{c}
n-1 \\
m+1
\end{array}\right|_{2}+\left.\left.n\right|_{m} ^{n-1}\right|_{2}\right) z^{-n-1} \\
& =\left.\left.\sum_{l=m}^{\infty}\right|_{m+1} ^{l}\right|_{2} z^{-l-2}-\sum_{n=m+1}^{\infty}\left|\begin{array}{c}
n-1 \\
m
\end{array}\right|_{2} \frac{\mathrm{d}}{\mathrm{d} z} z^{-n} \\
& =z^{-1} F_{m+1}(z)-F_{m}^{\prime}(z)
\end{aligned}
$$


and so

$$
F_{m+1}(z)=\frac{z}{1-z} F_{m}^{\prime}(z)=\cdots=\left(\frac{z}{1-z} \frac{\mathrm{d}}{\mathrm{d} z}\right)^{m} F_{1}(z) \quad(|z|>1) .
$$

For the final claim, note that, by (9), if $m \in \mathbb{N}$ and $|z|>1$ then

$$
\begin{aligned}
\sum_{n=1}^{N}\left|\begin{array}{c}
n \\
m
\end{array}\right|_{2}|z|^{-n-1} \frac{|w|^{m}}{m !} & \leqslant \sum_{n=m}^{N}\left(\begin{array}{c}
n \\
m
\end{array}\right)^{2} \frac{m}{n(n+1-m)}|z|^{-n-1}|w|^{m} \\
& \leqslant\left(\sum_{n=m}^{\infty}\left(\begin{array}{c}
n \\
m
\end{array}\right)|z|^{-(n+1) / 2}\right)^{2}|w|^{m}=\left(|z|^{1 / 2}-1\right)^{-2(m+1)}|w|^{m}
\end{aligned}
$$

for all $m \geqslant N$; the last step follows from (6). The result follows by the comparison test.

DeFinition 15. The Lambert $W$ function (also known as the product logarithm) is the multi-valued inverse to the map $w \mapsto w e^{w}$. Following the conventions of Corless et al. [8], the branch

$$
\widetilde{W}: z \mapsto\left\{\begin{array}{lll}
W_{-1}(z) & \text { if } z=-e^{-1}+r e^{i \theta} & (r>0, \theta \in] 0, \pi]) \\
W_{0}(z) & \text { if } z=-e^{-1}+r e^{i \theta} & (r \geqslant 0, \theta \in] \pi, 2 \pi])
\end{array}\right.
$$

is holomorphic on $\mathbb{C} \backslash\left[-e^{-1}, \infty\left[\right.\right.$ and has range containing $\Pi_{-}:=\{z \in \mathbb{C}: \operatorname{Im} z \in]-\pi, 0[\}$. TheOREM 16. If $X$ is a random variable with monotone Poisson distribution and mean $t>0$, the Cauchy transform of $X$ equals

$$
z \mapsto \frac{-1}{\widetilde{W}\left(-z e^{t-z}\right)} \quad \forall z \in \Pi_{+}:=\{z \in \mathbb{C}: \operatorname{Im} z \in] 0, \pi[\}
$$

where $\widetilde{W}$ is the branch of the Lambert $W$ function given in Definition 15.

Proof. The function

$$
\begin{aligned}
g: \operatorname{Dom} g:=\{x+i y: x \in \mathbb{R},|y|<\pi\} & \rightarrow\{x \cot x+i y:|y|<x<\pi\} \backslash[0,1[; \\
z & \mapsto-W_{0}\left(e^{z}\right)
\end{aligned}
$$

is a holomorphic bijection and

$$
g^{\prime}(z)=-e^{z} W_{0}^{\prime}\left(e^{z}\right)=\frac{-e^{z} e^{-W_{0}\left(e^{z}\right)}}{1+W_{0}\left(e^{z}\right)}=\frac{-W_{0}\left(e^{z}\right)}{1-\left(-W_{0}\left(e^{z}\right)\right)}=\frac{g(z)}{1-g(z)} \quad \forall z \in \operatorname{Dom} g .
$$

If $h(z):=z /(1-z)$ then $h \circ g=g^{\prime}$ and the chain rule implies that

$$
\frac{\mathrm{d}}{\mathrm{d} z} \circ c_{g}=m_{g^{\prime}} \circ c_{g} \circ \frac{\mathrm{d}}{\mathrm{d} z} \Leftrightarrow \frac{\mathrm{d}}{\mathrm{d} z} \circ c_{g}=c_{g} \circ m_{h} \circ \frac{\mathrm{d}}{\mathrm{d} z} \Leftrightarrow c_{g^{-1}} \circ \frac{\mathrm{d}}{\mathrm{d} z} \circ c_{g}=m_{h} \circ \frac{\mathrm{d}}{\mathrm{d} z},
$$

where $m_{h}: f \mapsto f h$ and $c_{g}: f \mapsto f \circ g$; the above holds when applied to any holomorphic function with domain including the range of $g$ (which does not include 0 or 1 ). Hence, by Lemma 14,

$$
F_{m}(z)=\left(\frac{z}{1-z} \frac{\mathrm{d}}{\mathrm{d} z}\right)^{m-1} \frac{1}{z(z-1)}=\left.\frac{\mathrm{d}^{m-1}}{\mathrm{~d} w^{m-1}} \frac{1}{g(w)(g(w)-1)}\right|_{w=g^{-1}(z)}=(1 / g)^{(m)}\left(g^{-1}(z)\right)
$$

for all $z \in \operatorname{Ran} g$ such that $|z|>1$; note that

$$
\frac{1}{g(z)(g(z)-1)}=-g^{\prime}(z) / g(z)^{2}=(1 / g)^{\prime}(z) \quad \forall z \in \text { Dom } g .
$$


Fix $z \in \operatorname{Ran} g$ such that $|z|^{1 / 2}>1+t^{1 / 2}$ and let $\varepsilon>0$ be such that $g^{-1}(z)+w \in \operatorname{Dom} g$ for all $w \in \mathbb{C}$ such that $|w|<\varepsilon$; suppose further that $\varepsilon^{1 / 2}<|z|^{1 / 2}-1$. By Taylor's theorem and Lemma 14, if $|w|<\varepsilon$ then

$$
\frac{1}{g\left(g^{-1}(z)+w\right)}=\frac{1}{z}+\sum_{m=1}^{\infty}(1 / g)^{(m)}\left(g^{-1}(z)\right) \frac{w^{m}}{m !}=\frac{1}{z}+\sum_{m=1}^{\infty} \sum_{n=1}^{\infty}\left|\begin{array}{l}
n \\
m
\end{array}\right|_{2} z^{-n-1} \frac{w^{m}}{m !} .
$$

As the first and last functions above are holomorphic functions of $w$ in

$$
\left\{w \in \mathbb{C}: g^{-1}(z)+w \in \operatorname{Dom} g\right\} \quad \text { and } \quad\left\{w \in \mathbb{C}:|w|^{1 / 2}<|z|^{1 / 2}-1\right\},
$$

respectively, and agree on $\{w \in \mathbb{C}:|w|<\varepsilon\}$, which lies in their intersection, they agree at $t$. Thus

$$
\frac{-1}{W_{0}\left(-z e^{t-z}\right)}=\frac{1}{g\left(g^{-1}(z)+t\right)}=\frac{1}{z}+\sum_{m=1}^{\infty} \sum_{n=1}^{\infty}\left|\begin{array}{c}
n \\
m
\end{array}\right|_{2} z^{-n-1} \frac{t^{m}}{m !}=G_{X}(z)
$$

for all $z \in \operatorname{Ran} g$ such that $|z|^{1 / 2}>1+t^{1 / 2}$, where the exchange of the order of summation which yields the last equality is a consequence of absolute convergence. Finally, $G_{X}$ is holomorphic on $\mathbb{C} \backslash \mathbb{R}$ and $z \mapsto-1 / \widetilde{W}\left(-z e^{t-z}\right)$ is holomorphic on $\Pi_{+}$; a short calculation shows that if $z \in \Pi_{+}$then $-z e^{t-z} \in \operatorname{Dom} \widetilde{W}$ and

$$
\frac{-1}{\widetilde{W}\left(-z e^{t-z}\right)}=\frac{-1}{W_{0}\left(-z e^{t-z}\right)} \quad \forall z \in \operatorname{Ran} g \cap\{z \in \mathbb{C}: \operatorname{Im} z>0\} .
$$

Since $G_{X}$ and $z \mapsto-1 / W_{0}\left(-z e^{t-z}\right)$ agree on $\left\{z \in \operatorname{Ran} g:|z|^{1 / 2}>1+t^{1 / 2}\right\}$ and this set meets $\Pi_{+}$, the result follows.

The following is contained in [16, Theorem 4.1] and may be proved, as it is there, by employing the Stieltjes inversion formula; the fact that the monotone Poisson distribution has an atom only at the origin will be useful later.

Corollary 17. If $X$ is a random variable distributed according to the monotone Poisson law with mean $t>0$ then

$$
\mathbb{P}(X \in A)=e^{-t} \delta_{0}(A)+\frac{1}{\pi} \int_{A \cap] a, b[} \operatorname{Im} \frac{1}{W_{-1}\left(-x e^{t-x}\right)} \mathrm{d} x
$$

for any measurable set $A \subseteq \mathbb{R}$, where $\delta_{0}$ is the Dirac measure which is supported at 0 , $a=-W_{0}\left(-e^{-(1+t)}\right)$ and $b=-W_{-1}\left(-e^{-(1+t)}\right)$.

5. The classical process. It is well known [3, Section 4.4] that monotone Brownian motion corresponds, via the isomorphism between quantum semimartingale algebras, to Azéma's martingale, i.e., the classical normal martingale $m=\left(m_{t}\right)_{t \geqslant 0}$ which satisfies the structure equation

$$
m_{0}=0, \quad \mathrm{~d}[m]_{t}=-m_{t-} \mathrm{d} m_{t}+\mathrm{d} t .
$$

A similar correspondence holds for the monotone Poisson process.

THEOREM 18. The quantum stochastic differential equation

$$
Y_{0}=0, \quad \mathrm{~d} Y=(I-Y) \mathrm{d} \Lambda+\mathrm{d} A+\mathrm{d} A^{\dagger}+\mathrm{d} t
$$


has a unique solution in the class of HP-adapted processes acting on exponential domains; this solution consists of commuting, self-adjoint operators such that $\left\|Y_{t}\right\| \leqslant(1+\sqrt{t})^{2}$ and, in the vacuum state, $Y_{t}$ obeys the monotone Poisson law with mean $t$, for all $t \geqslant 0$.

Proof. If $P=\left(P_{t}\right)_{t \geqslant 0}$ is the $\Omega$-adapted process such that

$$
P_{t}=\int_{0}^{t} \mathbb{E}_{s}\left(\mathrm{~d} \Lambda_{s}+\mathrm{d} A_{s}+\mathrm{d} A_{s}^{\dagger}+\mathrm{d} s\right) \quad \forall t \geqslant 0,
$$

where $\mathbb{E}$ is the conditional-expectation process in Boson Fock space, then $Y:=\hat{\pi}(P)$, the HP-adapted projection of $P$, satisfies (10) [3, Corollary 31]; alternatively, $Y$ can be viewed as the image of the Poisson process under Attal's $\mathcal{D}$ transform [2]. As $P$ is clearly bounded and self-adjoint, so is $Y$, and

$$
\left\|Y_{t}\right\|=\left\|P_{t}\right\| \leqslant\|\mathbb{E}\|_{\infty, t}+2\|\mathbb{E}\|_{2, t}+\|\mathbb{E}\|_{1, t}=1+2 \sqrt{t}+t \quad \forall t \geqslant 0 .
$$

Since $Y_{t}$ and $P_{t}$ have the same moments in the vacuum state, [4, Corollary 4.1] gives the claim about the distribution of $Y_{t}$.

For uniqueness, if $X$ is a solution of (10) which acts on some exponential domain $\mathcal{E}_{0}$ then

$$
(X-Y)_{0}=0, \quad \mathrm{~d}(X-Y)=-(X-Y) \mathrm{d} \Lambda \quad \text { on } \mathcal{E}_{0}
$$

as is well known, this equation admits only the zero process as a solution. Finally, if $s \geqslant 0$ is fixed and $X_{t}:=Y_{\min \{s, t\}}$ for all $t \geqslant 0$ then the quantum Itô formula yields

$$
\mathrm{d}(X Y-Y X)=-(X Y-Y X) \mathrm{d} \Lambda,
$$

so $(X Y-Y X)_{t}=0$ for all $t \geqslant 0$ and commutativity follows.

LEMMA 19. If $M$ and $N$ are regular quantum semimartingales and $t \geqslant 0$ then

$$
\int_{0}^{t} M_{s} \mathrm{~d} N_{s}=\lim _{n \rightarrow \infty} \sum_{i=1}^{n} M_{t_{i-1}^{n}}\left(N_{t_{i}^{n}}-N_{t_{i-1}^{n}}\right)
$$

strongly on exponential vectors, where $0=t_{0}^{n}<t_{1}^{n}<\cdots<t_{n}^{n}=t$ for all $n \geqslant 1$ are such that $\sup \left\{t_{i}^{n}-t_{i-1}^{n}: i=1, \ldots, n\right\} \rightarrow 0$ as $n \rightarrow \infty$.

Proof. If $\mathrm{d} N=E \mathrm{~d} \Lambda+F \mathrm{~d} A+G \mathrm{~d} A^{\dagger}+H \mathrm{~d} t, M_{t}^{(n)}:=M_{t_{i-1}^{n}}$ for $t \in\left[t_{i-1}^{n}, t_{i}^{n}\right.$ [ (where $i=1, \ldots, n)$ and $f \in L^{2}\left(\mathbb{R}_{+}\right)$then $[14, \operatorname{VI} .1(6.7),(7.4),(7.8)]$ there exists a constant $C_{f}$ such that, for all $t \geqslant 0$,

$$
\begin{aligned}
& \left\|\int_{0}^{t}\left(M_{s}-M_{s}^{(n)}\right) \mathrm{d} N_{s} \varepsilon(f)\right\|^{2} \\
& \leqslant C_{f} \int_{0}^{t}\left(|f(s)|^{2}\left\|\left(M_{s}-M_{s}^{(n)}\right) E_{s} \varepsilon(f)\right\|^{2}+\left\|\left(M_{s}-M_{s}^{(n)}\right) F_{s} \varepsilon(f)\right\|^{2}\right. \\
& \left.\quad+\left\|\left(M_{s}-M_{s}^{(n)}\right) G_{s} \varepsilon(f)\right\|^{2}\right) \mathrm{d} s+2\left(\int_{0}^{t}\left\|\left(M_{s}-M_{s}^{(n)}\right) H_{s} \varepsilon(f)\right\| \mathrm{d} s\right)^{2} \rightarrow 0
\end{aligned}
$$

as $n \rightarrow \infty$, by the dominated-convergence theorem; recall that $\mathbb{R}_{+} \ni t \mapsto M_{t}$ is strongly continuous on the whole of Fock space. The result follows because

$$
X \int_{s}^{t} \mathrm{~d} N_{r} \varepsilon(f)=\int_{s}^{t} X \mathrm{~d} N_{r} \varepsilon(f) \quad \forall X \in \mathcal{B}\left(\mathfrak{H}_{s}\right)
$$


if $0 \leqslant s \leqslant t$ (where $\mathfrak{H}_{s}$ denotes Boson Fock space up to time $s$ ); this is immediate when $E, F, G$ and $H$ are step functions and approximation with such yields the general case.

LEMma 20. If $(\Omega, \mathcal{F}, \mathbb{P})$ is a probability space, $\left(z_{t}: \Omega \rightarrow \mathbb{R}\right)_{t \geqslant 0}$ a family of bounded, $\mathcal{F}$-measurable functions and $\left(\mathcal{F}_{t}:=\sigma\left(z_{s}: 0 \leqslant s \leqslant t\right)\right)_{t \geqslant 0}$ their natural filtration then $\operatorname{alg}\left\{1, z_{s}: 0 \leqslant s \leqslant t\right\}$ is dense in $L^{2}\left(\mathcal{F}_{t}\right)$ for all $t \geqslant 0$.

Proof. If $f \in L^{2}\left(\mathcal{F}_{t}\right)$ then there exists $\left(t_{n}\right)_{n \geqslant 1}$ such that $f \in L^{2}\left(\sigma\left(z_{t_{n}}: n \geqslant 1\right)\right)$; let $\mathcal{G}_{n}:=\sigma\left(z_{t_{m}}: 1 \leqslant m \leqslant n\right)$ and note that $\mathbb{E}\left[f \mid \mathcal{G}_{n}\right] \rightarrow f$ as $n \rightarrow \infty$ (almost surely and in the $L^{2}$ sense). Hence it suffices to prove that $\mathfrak{A}_{n}:=\operatorname{alg}\left\{1, z_{t_{m}}: 1 \leqslant m \leqslant n\right\}$ is dense in $L^{2}\left(\mathcal{G}_{n}\right)$; fix $\left(\alpha_{1}, \ldots, \alpha_{n}\right) \in \mathbb{R}^{n}$ and let $\left(p_{k}\right)_{k \geqslant 1}$ be a sequence of polynomials such that $p_{k}\left(x_{1}, \ldots, x_{n}\right) \rightarrow \exp \left(-i\left(\alpha_{1} x_{1}+\cdots+\alpha_{n} x_{n}\right)\right)$ locally uniformly on $\mathbb{R}^{n}$. If $g \in L^{2}\left(\mathcal{G}_{n}\right)$ is orthogonal to $\mathfrak{A}_{n}$ then $g=h\left(z_{t_{1}}, \ldots, z_{t_{n}}\right)$ for some Borel-measurable function $h: \mathbb{R}^{n} \rightarrow \mathbb{C}$ and

$0=\mathbb{E}\left[g p_{k}\left(z_{t_{1}}, \ldots, z_{t_{n}}\right)\right] \rightarrow \mathbb{E}\left[g \exp \left(-i\left(\alpha_{1} z_{t_{1}}+\cdots+\alpha_{n} z_{t_{n}}\right)\right)\right]=\int_{\mathbb{R}^{n}} h(\mathbf{x}) \exp (-i \boldsymbol{\alpha} \cdot \mathbf{x}) \mathrm{d} \nu(\mathbf{x})$, where $\nu$ is the distribution of $\left(z_{t_{1}}, \ldots, z_{t_{n}}\right)$; the result follows from the injectivity of the Fourier transform.

THEOREM 21. There exists a martingale $x=\left(x_{t}\right)_{t \geqslant 0}$ (with respect to a filtration satisfying the usual conditions) which satisfies the structure equation

$$
x_{0}=0, \quad \mathrm{~d}[x]_{t}=\left(1-t-x_{t-}\right) \mathrm{d} x_{t}+\mathrm{d} t ;
$$

this process $x$ is unique in law and has the chaotic-representation property.

Proof. Identify $\mathfrak{A}$, the unital $C^{*}$ algebra generated by the family of operators $\left\{Y_{t}: t \geqslant 0\right\}$ from Theorem 18, with $C(\Delta)$, where $\Delta$ is a compact Hausdorff space, via the Gelfand map $A \mapsto \widehat{A}$. If $\Omega$ denotes the vacuum vector then $\widehat{A} \mapsto\langle\Omega, A \Omega\rangle$ is a positive linear functional on $C(\Delta)$ and the Riesz-Markov-Kakutani theorem yields a unique regular Borel measure $\mu$ on $\Delta$ such that

$$
\langle\Omega, A \Omega\rangle=\int_{\Delta} \widehat{A} \mathrm{~d} \mu \quad \forall A \in \mathfrak{A} ;
$$

since $\|\Omega\|=1, \mu$ is a probability measure. Thus if $X_{t}:=Y_{t}-t$ then $\left(x_{t}^{\circ}:=\widehat{X}_{t}\right)_{t \geqslant 0}$ is a family of square-integrable random variables on the probability space $(\Delta, \mathcal{B}, \mu)$ (where $\mathcal{B}$ is the Borel $\sigma$-algebra of $\Delta$ ).

Let $\mathcal{F}_{t}^{\circ}$ denote the $\sigma$-algebra generated by $\left\{x_{s}^{\circ}: 0 \leqslant s \leqslant t\right\}$, so that $\left(x_{t}^{\circ}\right)_{t \geqslant 0}$ is adapted to the filtration $\left(\mathcal{F}_{t}^{\circ}\right)_{t \geqslant 0}$. Since $\mathbb{E}_{s}\left(Y_{t}-t\right) \mathbb{E}_{s}=\left(Y_{s}-s\right) \mathbb{E}_{s}$ and $\mathbb{E}_{s} Y_{r} \mathbb{E}_{s}=Y_{r} \mathbb{E}_{s}$ for all $t \geqslant s \geqslant r \geqslant 0$, if $\widehat{Z}=z \in \operatorname{alg}\left\{1, x_{r}^{\circ}: 0 \leqslant r \leqslant s\right\}$ then $\mathbb{E}_{s} Z \mathbb{E}_{s}=Z \mathbb{E}_{s}$ and

$$
\int_{\Delta} \bar{z}\left(x_{t}^{\circ}-x_{s}^{\circ}\right) \mathrm{d} \mu=\left\langle Z \Omega,\left(Y_{t}-t-Y_{s}+s\right) \Omega\right\rangle=\left\langle Z \Omega, \mathbb{E}_{s}\left(Y_{t}-t-Y_{s}+s\right) \mathbb{E}_{s} \Omega\right\rangle=0
$$

if $t \geqslant s \geqslant 0$. Thus (by Lemma 20) $\mathbb{E}\left[x_{t}^{\circ} \mid \mathcal{F}_{s}^{\circ}\right]=x_{s}^{\circ}$ and $\left(x_{t}^{\circ}\right)_{t \geqslant 0}$ is a $\left(\mathcal{F}_{t}^{\circ}\right)_{t \geqslant 0}$-martingale such that $\mathbb{E}\left[\left(x_{t}^{\circ}\right)^{2}\right]=\left\langle\Omega, X_{t}^{2} \Omega\right\rangle=t$ for all $t \geqslant 0$, by the quantum Itô formula (or (1)). Furthermore, $\mathbb{E}\left[\left(x_{t}^{\circ}-x_{s}^{\circ}\right)^{2}\right]=t-s$ so $t \mapsto x_{t}^{\circ}$ is $L^{1}$-continuous; in particular, it has a modification $\left(x_{t}\right)_{t \geqslant 0}$ with càdlàg paths [18, Proposition II.67.6 and Theorem II.66.2] which is a martingale in the filtered probability space $\left(\Omega, \mathcal{F}, \mathbb{P},\left(\mathcal{F}_{t}\right)_{t \geqslant 0}\right)$, the $\mu$-completion 
of $(\Delta, \mathcal{B}, \mu)$ equipped with the usual augmentation of $\left(\mathcal{F}_{t}^{\circ}\right)_{t \geqslant 0}$ (which is right continuous and such that $\mathcal{F}_{0}$ is $\mathbb{P}$-complete) [18, Definition II.67.3].

The quantum Itô formula also implies that $X_{t}^{2}=X_{t}+\int_{0}^{t}\left(X_{s}-s\right) \mathrm{d} X_{s}+t$ for all $t \geqslant 0$, so if $z=\widehat{Z} \in \widehat{\mathfrak{A}}=C(\Delta)$ and $t \geqslant 0$ then, by Lemma 19 ,

$$
\begin{aligned}
\int_{\Delta} \bar{z}\left(x_{t}^{2}-x_{t}-t\right) \mathrm{d} \mathbb{P} & =\left\langle Z \Omega,\left(X_{t}^{2}-X_{t}-t\right) \Omega\right\rangle \\
& =\lim _{n \rightarrow \infty}\left\langle Z \Omega, \sum_{i=1}^{n}\left(X_{t_{i-1}^{n}}-t_{i-1}^{n}\right)\left(X_{t_{i}^{n}}-X_{t_{i-1}^{n}}\right) \Omega\right\rangle \\
& =\lim _{n \rightarrow \infty} \int_{\Delta} \bar{z} \sum_{i=1}^{n}\left(x_{t_{i-1}^{n}}-t_{i-1}^{n}\right)\left(x_{t_{i}^{n}}-x_{t_{i-1}^{n}}\right) \mathrm{d} \mathbb{P} \\
& =\int_{\Delta} \bar{z} \int_{0}^{t}\left(x_{s-}-s\right) \mathrm{d} x_{s} \mathrm{~d} \mathbb{P}
\end{aligned}
$$

since $C(\Delta)$ is dense in $L^{2}(\mathbb{P}), x_{t}^{2}-x_{t}-t=\int_{0}^{t}\left(x_{s-}-s\right) \mathrm{d} x_{s}$ and $\left(x_{t}\right)_{t \geqslant 0}$ is a normal martingale which satisfies the structure equation as claimed.

The other results follow from [9, Lemma 7] (with $f(t)=1-t$ and $g(s, t)=-1$ for all $s, t \geqslant 0)$, working as in [9, Proof of Proposition 6].

REMARK 22. The existence of a solution to (11) also follows from a much more general result of Taviot [20, Chapitre 4], which uses completely classical methods.

LEMMA 23. The martingale $x$ is purely discontinuous.

Proof. Note that, as $x_{t}^{c}=\int_{0}^{t} 1_{\left\{x_{s-}=1-s\right\}} \mathrm{d} x_{s}[9$, Proposition 1(ii)],

$$
\mathbb{E}\left[\left[x^{c}\right]_{t}\right]=\mathbb{E}\left[\int_{0}^{t} 1_{\left\{x_{s-}=1-s\right\}} \mathrm{d} s\right]=\int_{0}^{t} \mathbb{P}\left(x_{s}=1-s\right) \mathrm{d} s=0 ;
$$

this holds since $\left\{s: x_{s-}(\omega) \neq x_{s}(\omega)\right\}$ is countable almost surely and $\mathbb{P}\left(x_{s}=y\right)$ is non-zero if and only if $y=-s$ (note that $x_{s}+s$ is distributed according to the monotone Poisson law, which is atomic only at 0 by Corollary 17). Thus $x^{c} \equiv 0$, as claimed.

Theorem 24. If $\left(x_{t}\right)_{t \geqslant 0}$ is as in Theorem 21 and $y_{t}:=x_{t}+t$ then $\left(y_{t}\right)_{t \geqslant 0}$ is such that $\Delta y_{t}:=y_{t}-y_{t-} \in\left\{0,1-y_{t-}\right\}$ for all $t>0$. If

$$
\left.\left.g_{t}:=\sup \left\{s \in[0, t]: y_{s}=1\right\} \in\{-\infty\} \cup\right] 0, t\right]
$$

is the last time up to $t$ that $y$ hits 1 then

$$
y_{t}=-W_{\bullet}\left(-\exp \left(-1-t+g_{t}\right)\right) \quad \forall t \geqslant 0,
$$

where $W_{\bullet}$ equals $W_{0}$ or $W_{-1}$ (Definition 15$)$, so $y_{t} \in[0,1]$ or $y_{t} \in[1, \infty[$, respectively.

Proof. The statement about jumps follows from [9, Proposition 1(i)], or directly from the equation

$$
\mathrm{d}[y]_{t}=\left(1-y_{t-}\right) \mathrm{d} y_{t}+y_{t-} \mathrm{d} t
$$

this (and the identity $y_{t}^{2}=2 \int_{0}^{t} y_{s-} \mathrm{d} y_{s}+[y]_{t}$ ) also implies that

$$
\left(y_{t}-1\right)^{2}=1+2 \int_{0}^{t} y_{s-} \mathrm{d} s-[y]_{t} \quad \forall t \geqslant 0 .
$$


As (12) is clear for $t=0$, let $t>0$ and fix $\omega \in \Omega$ (which will be omitted in what follows, for the sake of brevity). If $g_{t}=-\infty$ then $y_{s} \neq 1$ for all $s \in[0, t]$, so $\Delta y_{s}=0$ for all $s \in] 0, t]$ and $y$ is continuous on $[0, t]$. As $x$ is purely discontinuous, by Lemma 23,

$$
[y]_{t}=[x]_{t}=\sum_{0<s \leqslant t} \Delta x_{s}^{2}=\sum_{0<s \leqslant t} \Delta y_{s}^{2}=0
$$

whence

$$
\left(y_{s}-1\right)^{2}=1+2 \int_{0}^{s} y_{r} \mathrm{~d} r \quad \forall s \in[0, t] .
$$

If $s \in[0, t]$ then $y_{s} \neq 1$, so $y_{r}+y_{s} \neq 2$ for all $r$ sufficiently near $s$ and

$$
\frac{y_{r}-y_{s}}{r-s}=\frac{\left(y_{r}-1\right)^{2}-\left(y_{s}-1\right)^{2}}{\left(y_{r}+y_{s}-2\right)(r-s)}=\frac{2}{\left(y_{r}+y_{s}-2\right)(r-s)} \int_{s}^{r} y_{u} \mathrm{~d} u \rightarrow \frac{y_{s}}{y_{s}-1}
$$

as $r \rightarrow s$. Hence $\mathrm{d} y_{s} / \mathrm{d} s=y_{s} /\left(y_{s}-1\right)$ for all $s \in[0, t]$ (taking one-sided derivatives at $s=0$ and $s=t$ ); from the initial condition $y_{0}=0$ it follows that $y \equiv 0$ on $[0, t]$, by Lemma 25, as required.

If $\left.\left.g_{t} \in\right] 0, t\right]$ then it is readily verified that $y_{g_{t}}=1$; if $g_{t}=t$ then (12) holds, so suppose that $g_{t}<t$. This implies that $y_{s} \neq 1$ for all $\left.\left.s \in\right] g_{t}, t\right]$ and $y$ is continuous on ]$\left.g_{t}, t\right]$; similar working to the above gives that $\mathrm{d} y_{s} / \mathrm{d} s=y_{s} /\left(y_{s}-1\right)$ for all $\left.\left.s \in\right] g_{t}, t\right]$, and the result follows from Lemma 25 (working on $\left[g_{t}+1 / n, t\right]$ and letting $n \rightarrow \infty$; note that $y_{g_{t}+1 / n}>0$, since $y_{g_{t}}=1$, for sufficiently large $n$ ).

LEMma 25. If $f:[s, t] \rightarrow \mathbb{R} \backslash\{1\}$ is continuously differentiable, with $f(s)=b \geqslant 0$ and $f^{\prime}(r)=f(r) /(f(r)-1)$ for all $r \in[s, t]$ (taking one-sided derivatives at $r=s$ and $r=t$ ), then

$$
f(r)=-W_{\bullet}(-b \exp (-r+s-b)) \quad \forall r \in[s, t],
$$

where $\bullet=0$ if $b \in[0,1[$ and $\bullet=-1$ if $b \in] 1, \infty[$.

Proof. Let $g(r)$ denote the right-hand side of (13); it is readily verified that $g(s)=b$, $g(r) \neq 1$ and $g^{\prime}(r)=g(r) /(g(r)-1)$ for all $r \in[s, t]$. If $c:=\sup \left\{|f(r)-1|^{-1}: s \leqslant r \leqslant t\right\}$ and $d:=\sup \left\{|g(r)-1|^{-1}: s \leqslant r \leqslant t\right\}$ then the fundamental theorem of calculus implies that

$$
\begin{aligned}
|(f-g)(r)| & \leqslant c d \int_{s}^{r}|(f-g)(u)| \mathrm{d} u \leqslant \cdots \\
& \leqslant \frac{(c d)^{n}(r-s)^{n}}{n !} \sup \{|(f-g)(u)|: s \leqslant u \leqslant r\} \rightarrow 0
\end{aligned}
$$

as $n \rightarrow \infty$, for all $r \in[s, t]$, which gives the result.

THEOREM 26. For all $h>0$ there exists a family of random variables $\left(a_{h, n}\right)_{n \in \mathbb{Z}_{+}}$such that $a_{h, 0}=0$,

$$
a_{h, n+1}^{2}=h+\left(1-n h-\sum_{k=0}^{n} a_{h, k}\right) a_{h, n+1} \quad \text { and } \quad \mathbb{E}\left[a_{h, n+1} \mid \sigma\left(a_{h, k}: 0 \leqslant k \leqslant n\right)\right]=0 .
$$

If $w_{h, t}:=\sum_{n=0}^{\lfloor t / h\rfloor} a_{h, n}$ for all $t \geqslant 0$, where $\lfloor s\rfloor$ denotes the greatest integer less than or equal to $s$, then $w_{h}:=\left(w_{h, t}\right)_{t \geqslant 0}$ is a martingale with respect to its natural filtration and 
satisfies

$$
w_{h, 0}=0, \quad \mathrm{~d}\left[w_{h}\right]_{t}=\left(1-t-w_{h, t-}\right) \mathrm{d} w_{h, t}+h \mathrm{~d}\lfloor t / h\rfloor .
$$

There exists a sequence $\left(h_{n}\right)_{n \geqslant 0}$ such that $\left(w_{h_{n}, t}\right)_{t \geqslant 0}$ converges weakly to the solution of the structure equation (11).

Proof. If such a family $\left(a_{h, n}\right)_{n \in \mathbb{Z}_{+}}$were to exist and $\phi_{n}:=\left(1-n h-w_{h, n h}\right) / 2$ then $w_{h,(n+1) h}=w_{h, n h}+\phi_{h, n}+\varepsilon_{h, n} \sqrt{\phi_{h, n}^{2}+h}$ for all $n \geqslant 0$, where

$$
\mathbb{P}\left(\varepsilon_{h, n}= \pm 1 \mid w_{h, n h}\right)=\frac{1}{2}\left(1 \mp \frac{\phi_{h, n}}{\sqrt{\phi_{h, n}^{2}+h}}\right),
$$

so $\left(w_{h, n h}\right)_{n \in \mathbb{Z}_{+}}$can be constructed as a non-homogeneous Markov process with these transition probabilities. The claims about $w_{h}$ are simple to verify; it is straightforward to check that

$$
w_{h, t}^{2}=w_{h, t}+\int_{0}^{t}\left(w_{h, s-}-s\right) \mathrm{d} w_{h, s}+\lfloor t / h\rfloor h \quad \forall t \geqslant 0 .
$$

Finally, that $\left(w_{h_{n}, t}\right)_{t \geqslant 0}$ converges to a solution of (11) for some $\left(h_{n}\right)_{n \geqslant 0}$ may be proved using the same techniques as Meyer [13].

REMARK 27. Using results of Attal [1] on representable probabilistic interpretations of Fock space, it can be shown that the classical solution constructed in Theorem 26 gives rise to the process that satisfies the quantum stochastic differential equation (10).

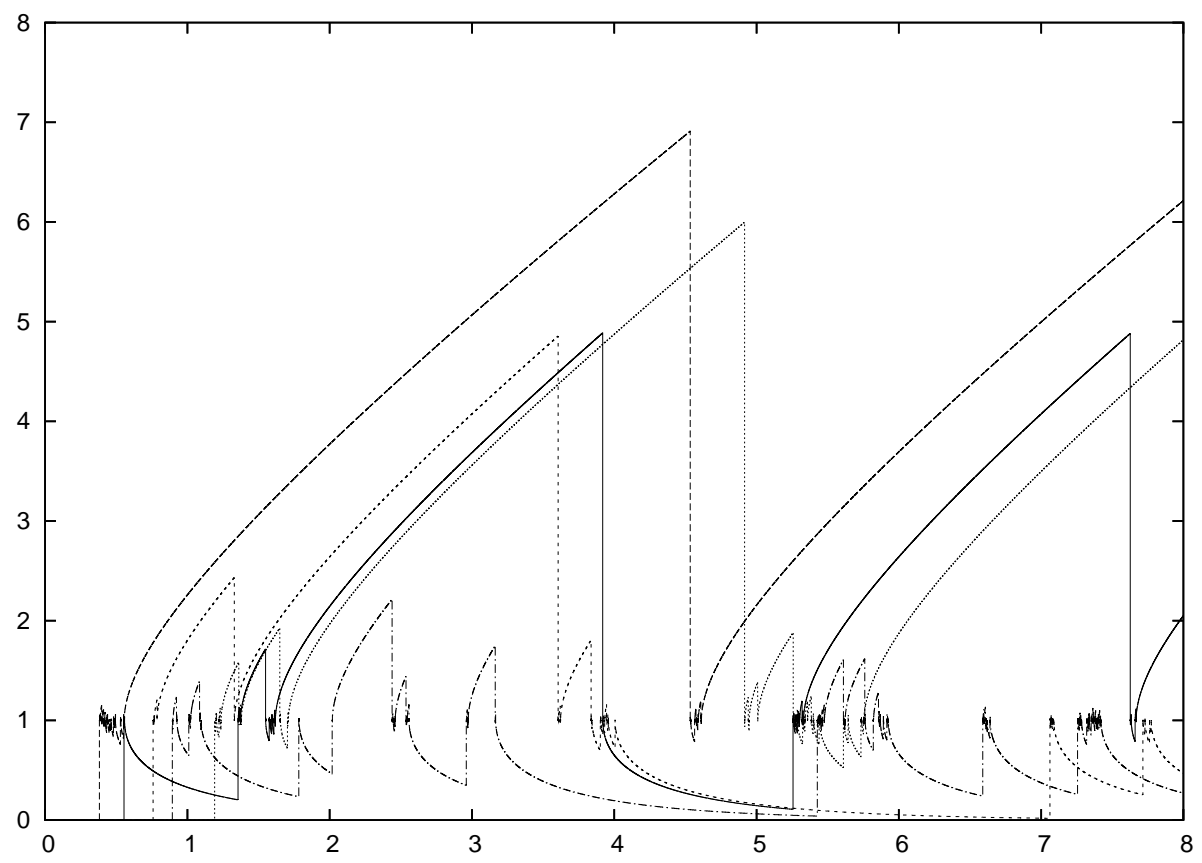

Fig. 1. Approximate sample paths of the monotone Poisson process 
REMARK 28. The calculation in the proof of Theorem 26 allows a numerical simulation to be performed: Figure 1 shows some sample paths of

$$
t \mapsto \tilde{y}_{h, t}:=\sum_{n=0}^{\lfloor t / h\rfloor} a_{h, n}+(t / h-\lfloor t / h\rfloor) a_{h,\lfloor t / h\rfloor+1}+t,
$$

with $h=0.005$ and $t \in[0,8[$.

Acknowledgements. The combinatorial part of this article had its genesis in a question from Dr Romuald Lenczewski at the 25th Conference on Quantum Probability and Infinite-Dimensional Analysis, Bȩdlewo, 2004; the hospitality of the organisers of that conference is gratefully acknowledged. Much of this work took place at Lady Margaret Hall, University of Oxford; the remainder was completed at IRMA, Strasbourg, where Prof. Michel Émery made some helpful comments, and the Université Claude Bernard Lyon 1, thanks to financial support provided through the European Community's Human Potential Programme under contract HPRN-CT-2002-00279, QP-Applications.

\section{References}

[1] S. Attal, An algebra of non-commutative bounded semimartingales: square and angle quantum brackets, J. Funct. Anal. 124 (1994), 292-332.

[2] S. Attal, The structure of the quantum semimartingale algebras, J. Operator Theory 46 (2001), 391-410.

[3] A. C. R. Belton, An isomorphism of quantum semimartingale algebras, Quart. J. Math. 55 (2004), 135-165.

[4] A. C. R. Belton, A note on vacuum-adapted semimartingales and monotone independence, in: Quantum Probability and Infinite Dimensional Analysis XVIII. From Foundations to Applications, M. Schürmann and U. Franz (eds.), World Scientific, Singapore, 2005, 105114.

[5] A. Z. Broder, The r-Stirling numbers, Discrete Math. 49 (1984), 241-259.

[6] L. Carlitz, Weighted Stirling numbers of the first and second kind. I, Fibonacci Quart. 18 (1980), 147-162.

[7] L. Carlitz, Weighted Stirling numbers of the first and second kind. II, Fibonacci Quart. 18 (1980), 242-257.

[8] R. M. Corless, G. H. Gonnet, D. E. G. Hare, D. J. Jeffrey and D. E. Knuth, On the Lambert $W$ function, Adv. Comput. Math. 5 (1996), 329-359.

[9] M. Émery, On the Azéma martingales, in: Séminaire de Probabilités XXIII, J. Azéma, P.-A. Meyer and M. Yor (eds.), Lecture Notes in Mathematics 1372, Springer, Berlin, 1989, 66-87.

[10] R. L. Graham, D. E. Knuth and O. Patashnik, Concrete Mathematics, second edition, Addison-Wesley, Massachusetts, 1994.

[11] H. Gupta, Selected Topics in Number Theory, Abacus Press, Tunbridge Wells, 1980.

[12] L. C. Hsu and P. J.-S. Shiue, A unified approach to generalized Stirling numbers, Adv. in Appl. Math. 20 (1998), 366-384.

[13] P.-A. Meyer, Construction de solutions d' "équations de structure", in: Séminaire de Probabilités XXIII, J. Azéma, P.-A. Meyer and M. Yor (eds.), Lecture Notes in Mathematics 1372, Springer, Berlin, 1989, 142-145. 
[14] P.-A. Meyer, Quantum Probability for Probabilists, second edition, Lecture Notes in Mathematics 1538, Springer, Berlin, 1995.

[15] D. S. Mitrinović, Sur une classe de nombres reliés aux nombres de Stirling, C. R. Acad. Sci. Paris 252 (1961), 2354-2356.

[16] N. Muraki, Monotonic independence, monotonic central limit theorem and monotonic law of small numbers, Infin. Dimens. Anal. Quantum Probab. Relat. Top. 4 (2001), 39-58.

[17] P. Protter, Stochastic Integration and Differential Equations, A New Approach, Applications of Mathematics 21, Springer, Berlin, 1990.

[18] L. C. G. Rogers and D. Williams, Diffusions, Markov Processes and Martingales. Volume 1: Foundations, second edition, Cambridge University Press, Cambridge, 2000.

[19] N. J. A. Sloane, The On-line Encyclopedia of Integer Sequences, http://www.research. att.com/ njas/sequences/, 2005.

[20] G. Taviot, Martingales et équations de structure : étude géométrique, doctoral thesis, Université Louis Pasteur Strasbourg 1, 1999. 
